\title{
An Inflammation-Index Signature Predicts Prognosis of Patients with Intrahepatic Cholangiocarcinoma After Curative Resection
}

\author{
Chaobin $\mathrm{He} \mathbb{D}^{1, *}$ \\ Chongyu Zhao',* \\ Yu Zhang ${ }^{2, *}$ \\ Cheng Chen ${ }^{3}$ \\ Xiaojun Lin $\mathbb{D}^{1}$
}

'Department of Pancreatobiliary Surgery, State Key Laboratory of Oncology in South China, Collaborative Innovation Center for Cancer Medicine, Sun Yat-sen University Cancer Center, Guangzhou, 510060, People's Republic of China;

${ }^{2}$ State Key Laboratory of Ophthalmology, Zhongshan Ophthalmic Center, Sun Yatsen University, Guangzhou, Guangdong, 510060, People's Republic of China; ${ }^{3}$ Department of Cardiology, The First Affiliated Hospital of Dalian Medical University, Dalian, People's Republic of China

*These authors contributed equally to this work
Correspondence: Xiaojun Lin Department of Pancreatobiliary Surgery, State Key Laboratory of Oncology in South China, Collaborative Innovation Center for Cancer Medicine, Sun Yat-sen University Cancer Center, Guangzhou, 510060, People's Republic of China

Email linxj@sysucc.org.cn
Background: The prognosis of patients with intrahepatic cholangiocarcinoma (ICC) after resection is at great variance. We aimed to establish a novel prognostic nomogram in facilitating the risk stratification for these patients.

Methods: A total of 82 high-dimensional radiological and pathological data were analyzed by LASSO-penalized Cox regression analyses and the panels with the best predictive performance were selected. Specific nomograms were established based on the selected panels and were validated in both primary $(n=292)$ and validation cohorts $(n=107)$. The area under the receiver operating characteristic curve (AUC) and the concordance index (C-index) were used to compare the predictive ability of nomograms and other staging systems.

Results: The modified Glasgow Prognostic Score (mGPS) was identified as the prognostic factor for both overall survival (OS) and progression-free survival (PFS). The nomograms built on the prognostic factors showed powerful efficacy in survival prediction, with C-indexes of 0.800 (95\% CI $0.767-0.833)$ and 0.752 (95\% CI $0.718-0.786)$ for OS and PFS in the primary cohort, 0.659 (95\% CI $0.586-0.732)$ and 0.638 (95\% CI $0.571-0.705)$ for OS and PFS in the validation cohort, respectively. Compared with tumor-node-metastasis stage, Barcelona Clinic Liver Cancer staging score, Cancer of the Liver Italian Program score, and Okuda staging system, the nomograms had significantly higher values of AUC and $\mathrm{C}$-indexes in survival prediction in the primary and validation cohorts.

Conclusion: Compared with currently used staging systems, the nomograms showed significantly higher efficacy in predicting survival of ICC patients after resection. The nomograms provide new versions of personalized management for these patients.

Keywords: intrahepatic cholangiocarcinoma, inflammation index, nomogram, prognosis, prediction

\section{Introduction}

Intrahepatic cholangiocarcinoma (ICC) is the second most common liver malignancy, ranking behind hepatocellular carcinoma (HCC) and its incidence continued to increase over the past two decades. ${ }^{1,2}$ Compared with HCC, ICC acted more aggressively and had a worse oncological outcome. ${ }^{3}$ Even after curative resection, the survival of ICC patients remains dismal. ${ }^{4}$

Accurate disease staging of ICC is critical for selecting proper treatment and predicting survival of patients. A wide variety of algorithms had been proposed in ICC for guiding treatment option and survival prediction, including tumor-node-metastasis 
(TNM) stage, Barcelona Clinic Liver Cancer (BCLC) staging score, Cancer of the Liver Italian Program (CLIP) score, and Okuda staging system. ${ }^{5-7}$ Besides, all these algorithms were based solely on pathological factors and mainly used for staging diseases, not survival prediction after resection. The ongoing increase in the incidence of ICC and the lack of knowledge of risk factors made it necessary to find a simple and effective prognostic system for patients with ICC after surgical resection.

As a remarkable hall-marker of cancer, inflammation component played an important role in the occurrence and development of tumors. ${ }^{8}$ Increasing evidence had shown that several inflammation-based indexes, including mGPS, ${ }^{9}$ NLR,${ }^{10}$ PLR, ${ }^{11}$ and PNI, ${ }^{12}$ correlated with cancer-specific survival in several kinds of cancers. These results suggested that inflammation-based indexes might be an important component for prognostic systems, apart from the pathological factors. Herein, we wished to establish prognostic nomograms based on inflammation-based indexes to predict survival for patients with ICC after radical resection and conduct a direct comparison of survival predictive power with currently available staging systems.

\section{Method}

\section{Patient Selection}

Consecutive patients who were pathologically diagnosed with ICC between January 2000 and December 2018 at the Sun Yat-sen University Cancer Center (SYSUCC) were enrolled to produce the primary cohort. The validation cohort was obtained from the First Affiliated Hospital of Dalian Medical University (FHDLMU) between May 2013 and December 2019. The same inclusion and exclusion

A

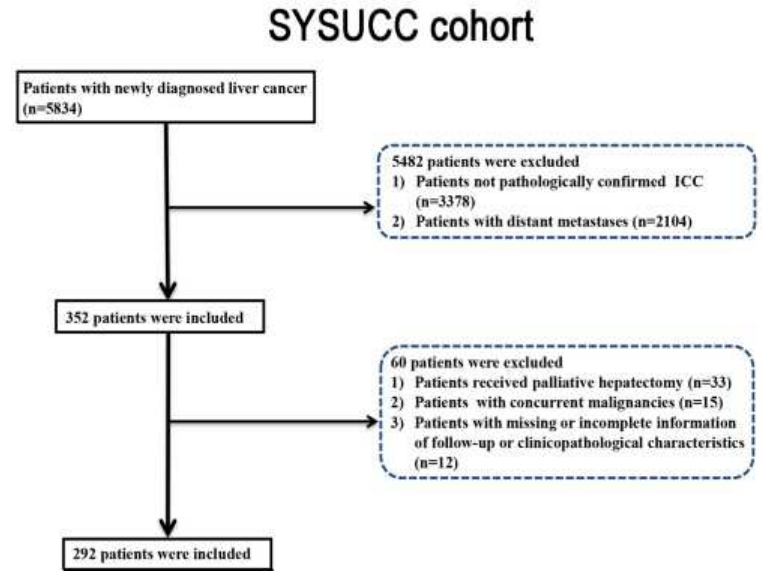

criteria were used for the enrollment of the primary and validation cohorts. Inclusion criteria were as follows: (1) age older than 18 years; (2) pathological confirmation of ICC; (3) eligible resection cases; (4) well-preserved liver function; (5) no other treatments before surgery. The following exclusion criteria were obtained: (1) evidence of hepatocellular decompensation, including refractory ascites, esophageal or gastric variceal bleeding, and hepatic encephalopathy; (2) obstructive jaundice; (3) concurrent primary cancers; (4) patients who were lost to follow-up; (5) patients whose clinical and pathological factors were missing. The flow diagram of the data selection is shown in Figure 1. This study was approved by the Institutional Review Board (IRB) of the SYSUCC and FHDLMU. Informed written consent was obtained from each individual participant. All procedures involving human participants were in accordance with the ethical standards of the 1964 Helsinki Declaration and its later amendments.

\section{Data Collection}

For all patients, detailed pathological variables of the specimens, which were described by experienced pathologist, were included into this study, including tumor size, tumor differentiation, LN metastasis, LN total number, satellite foci, macrovascular and microvascular invasion, lymph-vessel invasion, nerve tract invasion and adjacent organ invasion, pancreatic back membrane invasion. Additionally, the associated radiological and clinical variables were the same as those described in our previous study. ${ }^{13}$ The inflammation-based indexes, including neutrophil-to-lymphocyte ratio (NLR), platelet-to-lymphocyte ratio (PLR), lymphocyte-C-reactive protein ratio (LCR),

B

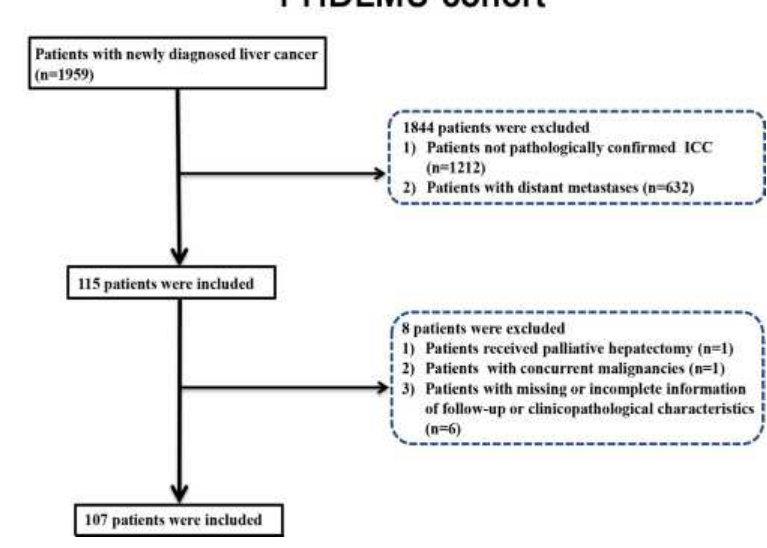

Figure I Flowchart of the included patients in the primary cohort $(\mathbf{A})$ and validation cohort (B). 
lymphocyte-to-monocyte ratio (LMR), modified Glasgow Prognostic Score (mGPS), prognostic nutritional index (PNI), prognostic index (PI), and systemic immuneinflammation index (SII), were included into this study. The description of these indexes was the same as that in our previous study. ${ }^{10}$ The optimal cut-off values for the inflammation indexes, including NLR, PLR, LCR, and LMR were determined using time-dependent ROC analysis. The NLR, PLR, LCR, and LMR scores were associated with the highest Youden index for the survival prediction, with these associated cutoff values. The threshold for each clinical and radiological dataset was utilized as the cut-off value for these variables.

\section{Surgical Procedure}

All included patients had received curative resection of tumors. Anatomic resection was used for hepatic lesions located in one segment. For multiple satellites located in more than one segment, nonanatomical resection was used for satellites with a negative resection margin, combined with the anatomical resection used for the main tumor. Nonanatomical resection was also used for patients with inadequate liver remnant. For suspected lymph node (LN) metastasis, those located within porta hepatis, hepatoduodenal ligament and retropancreatic and/or para-aortic were dissected as possible. Additionally, surgical resection was abandoned if metastatic retropancreatic or para-aortic LNs were pathologically confirmed by frozen-section examination, as the involvement of these LNs inhibited patients from benefiting from surgery. ${ }^{14}$

\section{Follow-Up}

The follow-up practice was finished at the outpatient clinic of SYSUCC and FHDLMU. Generally, follow-up strategies consisted of regular chest computed tomography (CT), abdominal CT, carbohydrate antigen 19-9 (CA199) and carcinoembryonic antigen (CEA) test at least every 3 months during the first year and every 6 months therefore. Occasional additional imaging modalities, such as positron emission tomography/CT, were selectively performed to determine patterns of recurrence. Follow-up data were retrieved at the end of November 2020. Progression-free survival (PFS) and overall survival (OS) were defined as the duration from the date of surgery until the date when tumor progression was diagnosed and death, respectively, or last follow-up.

\section{Statistical Analysis}

The categorical data, which were shown as frequencies and proportions, were compared with chi-square test. Log rank test was used to compare survival curves. The survival curves were generated using MedCalc software version 11.4.2.0 (http://www.medcalc.be). For OS and PFS prediction, multivariate Cox regression was conducted by least absolute shrinkage and selection operator (LASSO) logistic regression model based on the results of univariate analysis to identify significant prognostic factors. Nomograms were conducted with the prognostic factors according to previous protocols. ${ }^{15-17}$ Furthermore, the nomograms were further validated using the area under the receiver operating characteristic (ROC) curve (AUC) and concordance index (C-index). A two-tailed $\mathrm{P}<0.05$ was considered statistically significant. All statistical analyses were performed using $R$ version 3.6.1 software (The R Foundation for Statistical Computing, Vienna, Austria. http://www.r-project.org).

\section{Results}

\section{Patient Characteristics}

The demographic and clinical characteristics of the patients in the primary and validation cohorts are shown in Table 1. A total of 292 patients from SYSUCC that served as primary cohort and another 107 patients from FHDLMU that served as validation cohort were included in this study. For the primary cohort, $181(62.0 \%)$ patients were male and $111(38 \%)$ patients were female. A total of $189(64.7 \%)$ patients were younger than 60 years and the remaining $103(35.3 \%)$ patients were older than 60 years old. The median age was 56 years (range, 20-77 years). Tumors of most patients $(177,60.6 \%)$ were larger than $5 \mathrm{~cm}$ and a total of $131(44.9 \%)$ patients had received chemotherapy after resection. As for validation cohort, most of patients were male and were older than 60 years old. A total of $55(51.4 \%)$ patients had tumors which were larger than $5 \mathrm{~cm}$ while tumors in a small portion of patients were with vascular invasion. Thirty-five (32.7\%) patients had received chemotherapy after resection.

\section{Independent Prognostic Factors in the Primary Cohort}

The median OS of patients were 39.47 months $(95 \% \mathrm{CI}$, 31.03-49.87 months) in the SYSUCC cohort, and 16.23 months (95\% CI, 12.23-24.10 months) in the FHDMU cohort, respectively. The median PFS was 11.23 months (95\% CI, 8.87-14.13 months) in the SYSUCC cohort, and 
Table I Clinicopathological and Radiological Characteristics of Primary and Validation Cohorts

\begin{tabular}{|c|c|c|}
\hline Variables & $\begin{array}{l}\text { Primary Cohort } \\
(n=292)\end{array}$ & $\begin{array}{l}\text { Validation Cohort } \\
(n=107)\end{array}$ \\
\hline \multicolumn{3}{|l|}{ Gender } \\
\hline Male & I8I (62.0\%) & 62 (57.9\%) \\
\hline Female & III (38.0\%) & $45(42.1 \%)$ \\
\hline \multicolumn{3}{|c|}{ Age (years) } \\
\hline$\leq 60$ & I 89 (64.7\%) & 33 (30.8\%) \\
\hline$>60$ & $103(35.3 \%)$ & $74(69.2 \%)$ \\
\hline \multicolumn{3}{|c|}{ WBC count $\left(\times 10^{9} / \mathrm{L}\right)$} \\
\hline$\leq 10$ & 259 (88.7\%) & 92 (86.0\%) \\
\hline$>10$ & $33(11.3 \%)$ & $15(14.0 \%)$ \\
\hline \multicolumn{3}{|c|}{ HGB (g/L) } \\
\hline$\leq 120$ & 27 (9.20\%) & $30(28.0 \%)$ \\
\hline$>120$ & $265(90.8 \%)$ & 77 (72.0\%) \\
\hline \multicolumn{3}{|c|}{ PLT $\left(\times 10^{9} / L\right)$} \\
\hline$\leq 100$ & $10(3.40 \%)$ & $5(4.70 \%)$ \\
\hline$>100$ & $282(96.6 \%)$ & $102(95.3 \%)$ \\
\hline \multicolumn{3}{|l|}{ ALT (U/L) } \\
\hline$\leq 40$ & $236(80.8 \%)$ & 55 (5I.4\%) \\
\hline$>40$ & $56(19.2 \%)$ & $52(48.6 \%)$ \\
\hline \multicolumn{3}{|l|}{ AST (U/L) } \\
\hline$\leq 45$ & $254(87.0 \%)$ & $56(52.3 \%)$ \\
\hline$>45$ & $38(13.0 \%)$ & $5 \mathrm{I}(47.7 \%)$ \\
\hline \multicolumn{3}{|c|}{ GGT (U/L) } \\
\hline$\leq 50$ & 108 (37.0\%) & $16(15.0 \%)$ \\
\hline$>50$ & I84 (63.0\%) & 91 (85.0\%) \\
\hline \multicolumn{3}{|c|}{ ALP (U/L) } \\
\hline$\leq 100$ & $182(62.3 \%)$ & $25(23.4 \%)$ \\
\hline$>100$ & 110 (37.7\%) & $82(76.6 \%)$ \\
\hline \multicolumn{3}{|l|}{ ALB $(g / L)$} \\
\hline$\leq 40$ & 5 (I.70\%) & 38 (35.5\%) \\
\hline$>40$ & 287 (98.3\%) & 69 (64.5\%) \\
\hline \multicolumn{3}{|c|}{ TBIL ( $\mu \mathrm{mol} / \mathrm{L})$} \\
\hline$\leq 20.5$ & 265 (90.8\%) & 54 (50.5\%) \\
\hline$>20.5$ & 27 (9.20\%) & $53(49.5 \%)$ \\
\hline \multicolumn{3}{|c|}{ IBIL $(\mu \mathrm{mol} / \mathrm{L})$} \\
\hline$\leq 15$ & 275 (94.2\%) & $65(60.7 \%)$ \\
\hline$>15$ & 17 (5.80\%) & 42 (39.3\%) \\
\hline
\end{tabular}

(Continued)
Table I (Continued).

\begin{tabular}{|c|c|c|}
\hline Variables & $\begin{array}{l}\text { Primary Cohort } \\
(n=292)\end{array}$ & $\begin{array}{l}\text { Validation Cohort } \\
(n=107)\end{array}$ \\
\hline \multicolumn{3}{|c|}{ CRP (mg/L) } \\
\hline$\leq 3$ & I 72 (58.9\%) & 35 (32.7\%) \\
\hline$>3$ & 120 (4I. I\%) & 72 (67.3\%) \\
\hline \multicolumn{3}{|l|}{ HBsAg } \\
\hline Absence & 162 (55.5\%) & 105 (98.1\%) \\
\hline Presence & I 30 (44.5\%) & $2(1.9 \%)$ \\
\hline \multicolumn{3}{|c|}{ CA I 9-9 (U/mL) } \\
\hline$\leq 35$ & |4| (48.3\%) & 25 (23.4\%) \\
\hline$>35$ & I5I (5I.7\%) & 82 (76.6\%) \\
\hline \multicolumn{3}{|c|}{ CEA (ng/mL) } \\
\hline$\leq 5$ & 211 (72.3\%) & $60(56.1 \%)$ \\
\hline$>5$ & 81 (27.7\%) & 47 (43.9\%) \\
\hline \multicolumn{3}{|l|}{ LCR } \\
\hline 0 & 21 (7.20\%) & - \\
\hline I & $27 \mid(92.8 \%)$ & - \\
\hline \multicolumn{3}{|l|}{ mGPS } \\
\hline 0 & $216(74.0 \%)$ & 37 (34.6\%) \\
\hline I & 67 (22.9\%) & 43 (40.2\%) \\
\hline 2 & $9(3.10 \%)$ & 27 (25.2\%) \\
\hline \multicolumn{3}{|l|}{ NLR } \\
\hline$<2.62$ & 194 (66.4\%) & 36 (33.6\%) \\
\hline$\geq 2.62$ & 98 (33.6\%) & 71 (66.4\%) \\
\hline \multicolumn{3}{|l|}{ LMR } \\
\hline$<4.06$ & $125(42.8 \%)$ & - \\
\hline$\geq 4.06$ & 167 (57.2\%) & - \\
\hline \multicolumn{3}{|l|}{ PLR } \\
\hline$<104.85$ & I 72 (58.9\%) & 24 (22.4\%) \\
\hline$\geq 104.85$ & 120 (4I.1\%) & 83 (77.6\%) \\
\hline \multicolumn{3}{|l|}{ SII } \\
\hline 0 & $68(23.3 \%)$ & 30 (28.0\%) \\
\hline I & 224 (76.7\%) & 77 (72.0\%) \\
\hline \multicolumn{3}{|l|}{ PNI } \\
\hline 0 & 277 (94.9\%) & 48 (44.9\%) \\
\hline 1 & $15(5.1 \%)$ & 59 (55.1\%) \\
\hline \multicolumn{3}{|l|}{ PI } \\
\hline 0 & 220 (75.3\%) & 32 (29.9\%) \\
\hline I & 61 (20.9\%) & $63(58.9 \%)$ \\
\hline
\end{tabular}

(Continued) 
Table I (Continued).

\begin{tabular}{|c|c|c|}
\hline Variables & $\begin{array}{l}\text { Primary Cohort } \\
(n=292)\end{array}$ & $\begin{array}{l}\text { Validation Cohort } \\
(n=107)\end{array}$ \\
\hline 2 & II (3.8\%) & $12(11.2 \%)$ \\
\hline \multicolumn{3}{|c|}{ Tumor capsular } \\
\hline Absence & 45 (I5.4\%) & - \\
\hline Uncompleted & 37 (12.7\%) & - \\
\hline Completed & $210(71.9 \%)$ & - \\
\hline \multicolumn{3}{|l|}{ Satellite foci } \\
\hline Absence & 201 (68.8\%) & $106(99.1 \%)$ \\
\hline Presence & 91 (3I.2\%) & I (0.90\%) \\
\hline \multicolumn{3}{|c|}{ Vascular thrombus } \\
\hline Absence & $269(92.1 \%)$ & - \\
\hline Presence & $23(7.90 \%)$ & - \\
\hline \multicolumn{3}{|c|}{ Tumor differentiation } \\
\hline Low & $6(2.10 \%)$ & $3(2.80 \%)$ \\
\hline Medium & 105 (35.9\%) & 81 (75.7\%) \\
\hline High & I8I (62.0\%) & $23(21.5 \%)$ \\
\hline \multicolumn{3}{|c|}{ Microvascular invasion } \\
\hline Absence & 237 (81.2\%) & 86 (89.7\%) \\
\hline Presence & $55(18.8 \%)$ & II (10.3\%) \\
\hline \multicolumn{3}{|c|}{ Lymph-vessel invasion } \\
\hline Absence & 273 (93.5\%) & - \\
\hline Presence & $19(6.5 \%)$ & - \\
\hline \multicolumn{3}{|c|}{ Macrovascular invasion } \\
\hline Absence & 274 (93.8\%) & 95 (88.8\%) \\
\hline Presence & $18(6.20 \%)$ & $12(11.2 \%)$ \\
\hline \multicolumn{3}{|c|}{ Back membrane invasion } \\
\hline Absence & II 4 (39.0\%) & 90 (84.1\%) \\
\hline Presence & $178(61.0 \%)$ & $12(15.9 \%)$ \\
\hline \multicolumn{3}{|c|}{ Imaging tumor size } \\
\hline$\leq 5 \mathrm{~cm}$ & I3I (44.9\%) & $56(52.3 \%)$ \\
\hline$>5 \mathrm{~cm}$ & $161(55.1 \%)$ & $5 \mathrm{I}(47.7 \%)$ \\
\hline \multicolumn{3}{|c|}{ Imaging vascular invasion } \\
\hline Absence & 271 (92.8\%) & $97(90.7 \%)$ \\
\hline Presence & 21 (7.20\%) & $10(9.30 \%)$ \\
\hline \multicolumn{3}{|c|}{ Imaging LN metastasis } \\
\hline Absence & 207 (70.9\%) & $54(50.5 \%)$ \\
\hline Presence & $85(29.1 \%)$ & $53(49.5 \%)$ \\
\hline
\end{tabular}

(Continued)
Table I (Continued).

\begin{tabular}{|l|l|l|}
\hline Variables & $\begin{array}{l}\text { Primary Cohort } \\
(n=292)\end{array}$ & $\begin{array}{l}\text { Validation Cohort } \\
(n=107)\end{array}$ \\
\hline
\end{tabular}

\section{Imaging LN size}

\begin{tabular}{l|l|l}
\hline Absence & $207(70.9 \%)$ & - \\
$\leq \mathrm{Icm}$ & $28(9.60 \%)$ & - \\
$>\mathrm{Icm}$ & $57(19.5 \%)$ & - \\
\hline
\end{tabular}

Tumor size
\begin{tabular}{l|l|l|}
$\leq 5 \mathrm{~cm}$ & $115(39.4 \%)$ & $52(48.6 \%)$ \\
$>5 \mathrm{~cm}$ & $177(60.6 \%)$ & $55(51.4 \%)$
\end{tabular}

\section{LN metastasis}

\begin{tabular}{|l|l|l|}
\hline Absence & $250(85.6 \%)$ & $95(88.8 \%)$ \\
Presence & $42(14.4 \%)$ & $12(11.2 \%)$ \\
\hline
\end{tabular}

\section{Nerve tract invasion}

\begin{tabular}{|l|l|l|}
\hline Absence & $96(89.7 \%)$ & $96(89.7 \%)$ \\
Presence & $11(10.3 \%)$ & $11(10.3 \%)$ \\
\hline
\end{tabular}

Adjacent origin invasion

\begin{tabular}{|c|c|c|}
\hline $\begin{array}{l}\text { Absence } \\
\text { Presence }\end{array}$ & $\begin{array}{l}269(92.1 \%) \\
23(7.90 \%)\end{array}$ & $\begin{array}{l}103(96.3 \%) \\
4(3.70 \%)\end{array}$ \\
\hline \multicolumn{3}{|c|}{ T stage 8th } \\
\hline I & 34 (24.3\%) & 84 (78.5\%) \\
\hline 2 & 44 (15.1\%) & $5(4.7 \%)$ \\
\hline 3 & 153 (52.4\%) & 14 (13.1\%) \\
\hline 4 & 24 (8.20\%) & $4(3.7 \%)$ \\
\hline \multicolumn{3}{|c|}{ N stage 8th } \\
\hline Absence & 250 (85.6\%) & 95 (88.8\%) \\
\hline Presence & 42 (14.4\%) & $12(11.2 \%)$ \\
\hline \multicolumn{3}{|l|}{ TNM 8th } \\
\hline 1 & 70 (24.0\%) & 81 (75.7\%) \\
\hline II & 37 (12.7\%) & $2(1.90 \%)$ \\
\hline III & I85 (63.4\%) & 24 (22.4\%) \\
\hline \multicolumn{3}{|c|}{ Chemotherapy } \\
\hline Absence & $16 \mid(55.1 \%)$ & 72 (67.3\%) \\
\hline Presence & $|3|$ (44.9\%) & 35 (32.7\%) \\
\hline
\end{tabular}

Abbreviations: WBC, white blood cell count; HGB, hemoglobin; PLT, platelets; ALT, alanine aminotransferase; AST, aspartate aminotransferase; ALB, albumin; TBIL, total serum bilirubin; IBIL, indirect serum bilirubin; CRP, C-reactive protein; $\mathrm{HBs} A g$, hepatitis B virus antigen; CA19-9, carbohydrate antigen 19-9; CEA, carcinoembryonic antigen; LCR, lymphocyte-to-CRP ratio; mGPS, modified Glasgow Prognostic Score; NLR, neutrophil-to-lymphocyte ratio; LMR, lymphocyte-tomonocyte ratio; PLR, platelet-lymphocyte ratio; SIl, systemic immuneinflammation index; PNI, prognostic nutritional index; PI, prognostic index; LN, lymph node metastasis; TNM, tumor-node-metastasis. 
12.87 months (95\% CI, 10.10-16.97 months) in the FHDMU cohort, respectively. In order to investigate prognostic factors for OS and PFS, LASSO-penalized Cox regression analyses were performed based on 82 highdimensional radiological and clinicopathological data to further reduce the number of factors in the selected panel with the best predictive performance using 10-fold cross validation. Seven variables were selected for OS prediction by the LASSO-Cox regression model, including mGPS [hazard ratio $(\mathrm{HR})=10.21,95 \% \mathrm{CI} 9.87-10.55, \mathrm{P}<$ $0.001]$, ALP $(\mathrm{HR}=1.07,95 \%$ CI 1.01-1.13, $\mathrm{P}=0.045)$, CA19-9 $(\mathrm{HR}=2.23,95 \%$ CI 1.88-2.58, $\mathrm{P}=0.026), \mathrm{CEA}$ $(\mathrm{HR}=2.78,95 \% \mathrm{CI} 2.41-3.15, \mathrm{P}=0.005)$, tumor number $(\mathrm{HR}=2.10,95 \%$ CI 1.76-2.44, $\mathrm{P}=0.036)$, tumor size (HR $=2.22,95 \% \mathrm{CI} 1.80-2.64, \mathrm{P}=0.027)$, and $\mathrm{LN}$ metastasis $(\mathrm{HR}=2.07,95 \%$ CI 1.64-2.49, $\mathrm{P}=0.039)($ Figures $2 \mathrm{~A}$ and
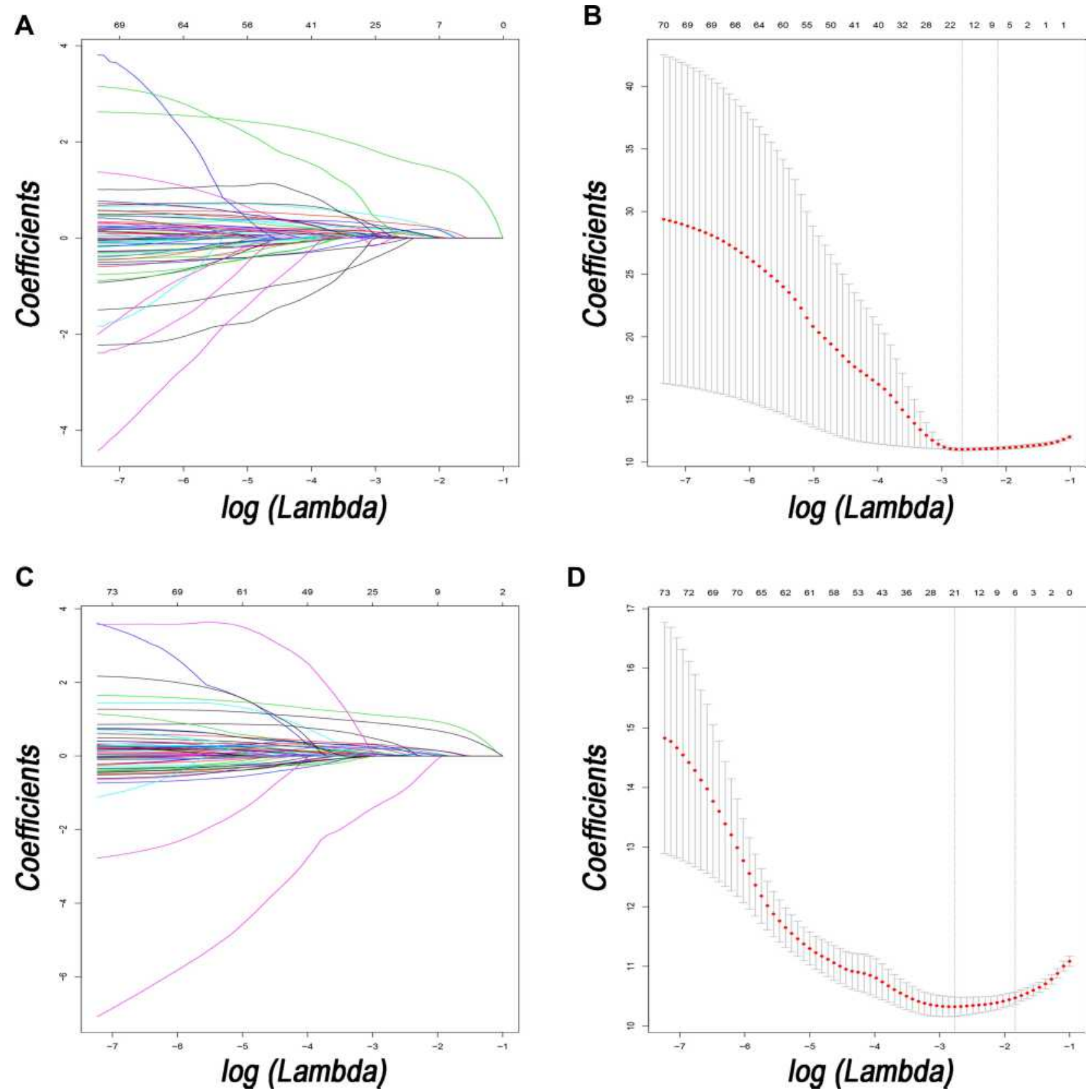

D

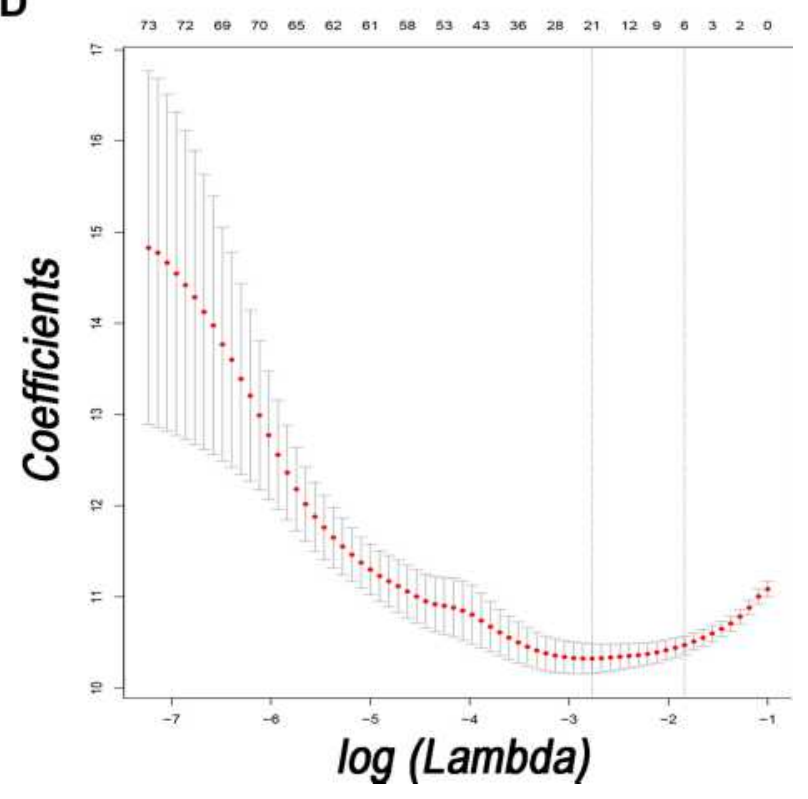

Figure 2 Feature selection using the least absolute shrinkage and selection operator (LASSO) Cox regression model. LASSO coefficient profiles of 82 variables against the $\log$ (Lambda) sequence for OS (A) and tuning parameter (Lambda) selection in the LASSO model used I0-fold cross-validation via minimum criteria for OS (B). LASSO coefficient profiles of 82 variables against the log (Lambda) sequence for PFS (C) and tuning parameter (Lambda) selection in the LASSO model used I0-fold cross-validation via minimum criteria for PFS (D).

Abbreviations: OS, overall survival; PFS, progression-free survival. 
B). In terms of PFS, the association between these factors and survival was also explored with LASSO-penalized Cox regression analysis. It was shown that $\mathrm{mGPS}(\mathrm{HR}=9.01$, 95\% CI 8.73-9.29, $\mathrm{P}<0.001)$, imaging tumor size $(\mathrm{HR}=$ $1.41,95 \%$ CI $1.06-1.76, \mathrm{P}=0.047)$, tumor number $(\mathrm{HR}=$ $1.91,95 \%$ CI $1.60-2.22, \mathrm{P}=0.045)$, tumor size $(\mathrm{HR}=2.12$, $95 \%$ CI 1.74-2.50, $\mathrm{P}=0.034)$, and chemotherapy $(\mathrm{HR}=$ $6.84,95 \%$ CI $6.53-7.15, \mathrm{P}<0.001)$ were identified as independent prognostic factors for PFS (Figure $2 \mathrm{C}$ and D).

\section{Construction and Validation of the Nomograms}

The prognostic nomograms which were constructed based on the selected prognostic factors were shown in Figure 3. The prognostic factor, mGPS, demonstrated the most prominent effect in both of OS and PFS prediction. The calibration plots for the probability of OS and PFS at 1-, 2-, and 3-year for primary cohort showed good agreement between the prediction by nomograms and the actual observations (Figure 4A and B). For the prediction of survival in validation cohort, the calibration plots for the probability of OS and PFS at 1-, 2-, and 3-year showed optimal consistency between prediction by nomograms and the actual observation (Figure 4C and D).

\section{Comparative Performances of the Predictive Nomograms}

The predictive capability of the prognostic nomogram and the staging systems were compared in terms of the C-index and AUC. In terms of OS prediction, the C-indexes of the nomograms were 0.800 (95\% CI 0.767-0.833) and 0.659 (95\% CI $0.586-0.732)$ for the primary and validation cohorts, respectively. The established nomogram possessed the largest $\mathrm{C}$-indexes relative to TNM 8th edition, BCLC, CLIP, and Okuda staging systems (C-index comparison for OS prediction: nomogram vs TNM 8th edition, $p<0.001$ for primary cohort and $\mathrm{P}=0.003$ for validation cohort; nomogram vs BCLC, nomogram vs CLIP, nomogram vs Okuda staging system, all $p<0.001$ for both primary and validation cohorts, Table 2). Additionally, compared with other staging systems, the AUC of nomogram was the largest, which indicated that the nomogram to be a superior predictive model (Figure 5).

In terms of PFS prediction, the nomograms for PFS prediction also showed relatively high predictive power with the C-indexes of 0.752 (95\% CI $0.718-0.786)$ and
0.638 (95\% CI $0.571-0.705)$ for the primary and validation cohorts, respectively. Similarly, the established nomogram possessed the significantly higher C-indexes, compared with those of TNM 8th edition, BCLC, CLIP, and Okuda staging systems (Table 2). The results of AUC comparisons also consolidated the stronger predictive power of the established nomogram, compared with other common staging systems (Figure S1).

\section{Discrimination Ability of the Prognostic Nomograms}

Based on the survival risk of different scores, patients were grouped into high- and low-risk groups, stratified by the median values of the nomogram scores. The 1-, 2and 3-year OS rates of low and high-risk groups were $96.5 \%, 88 \%, 76.8 \%$ and $58.3 \%, 40.6 \%, 23.2 \%$, respectively, for primary cohort $(\mathrm{P}<0.001)$. For validation cohort, compared with those of high-risk groups, patients in the low-risk groups also possessed significantly higher OS rates, with the 1-, 2- and 3-year OS rates of 71.6\%, $50.9 \%$, and $45.5 \%$, respectively $(\mathrm{P}=0.001)$. Similarly, in terms of PFS, higher survival rates were also observed in patients with low nomogram-based risks, compared with those with high nomogram-based risks (Figure 6). The results revealed that the established nomograms were useful predictors for the survival of ICC patients after resection in both primary and validation cohorts.

\section{Discussion}

Patients with ICC are frequently associated with a shortterm survival time and relatively high rates of recurrence after the initial resection, mainly due to the relatively late diagnosis, local invasion, and distant metastasis. ${ }^{18}$ Considering the fact that systemic inflammatory response could also affect survival, additional modifications of the pathological variables-based staging systems need to be incorporated to help better refine the prognostic stratification of patients with ICC.

A tight cross-talk was developed between tumor cells and tumor microenvironment, which was regulated by relevant cytokines and signal transduction. ${ }^{19}$ Previous studies had illustrated that inflammation contributed to bile duct carcinoma. ${ }^{20,21}$ Inflammation mediators are the hallmark of several risk factors associated with ICC. ${ }^{22} \mathrm{We}$ included several inflammation-based scores and analyzed their prognostic efficacy in this large, multicentre cohort study. It was shown that mGPS was identified as the 
A

Points

mGPS

Tumor number

ALP

CA19-9

CEA

Tumor size

LN metastasis

Total points

Linear Predictor

1-year OS rate

2-year OS rate

3-year OS rate

B

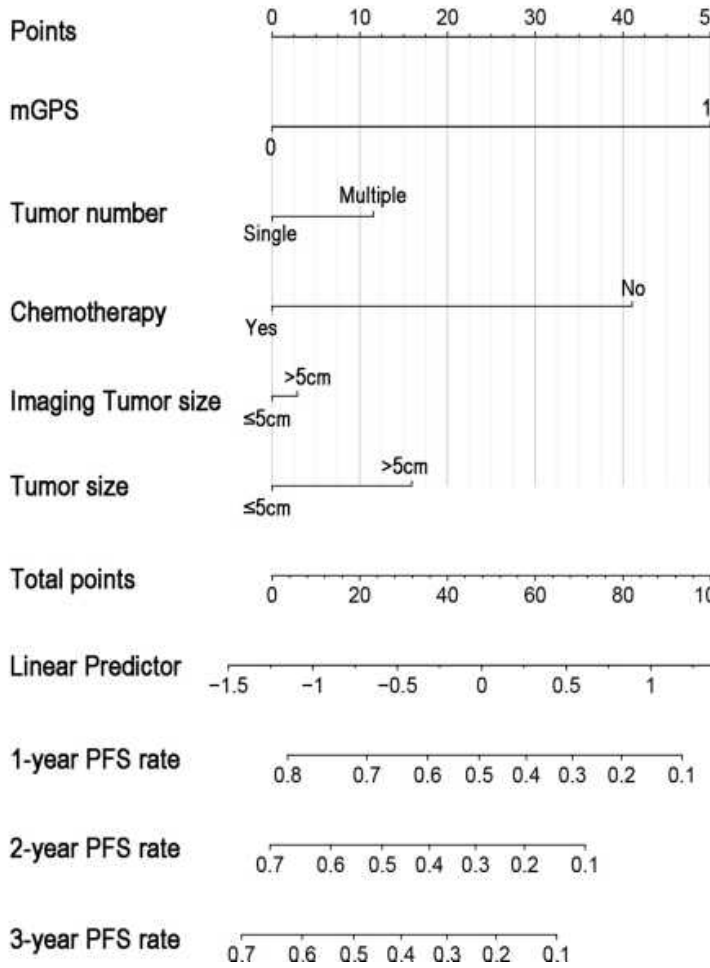

$\begin{array}{lllllllllll}0.9 & & 0.8 & 0.7 & 0.6 & 0.5 & 0.4 & 0.3 & 0.2 & 0.1\end{array}$

\section{$\begin{array}{lllll}0.8 & 0.7 & 0.6 & 0.50 .40 .30 .20 .1\end{array}$}

$\begin{array}{lllllllllll}0 & 10 & 20 & 30 & 40 & 50 & 60 & 70 & 80 & 90 & 100\end{array}$
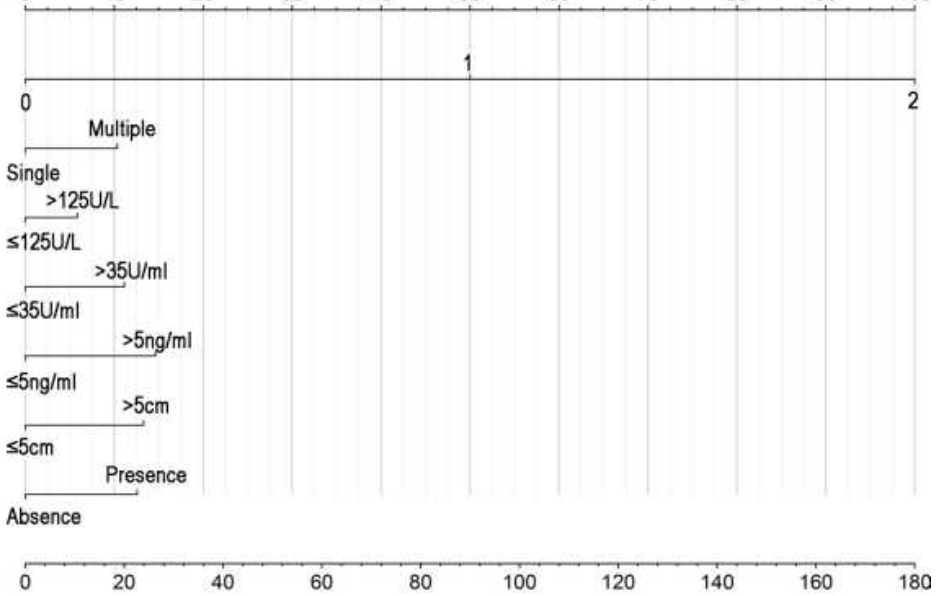

$\begin{array}{lllllllllllll}-1.5 & -1 & -0.5 & 0 & 0.5 & 1 & 1.5 & 2 & 2.5 & 3 & 3.5 & 4 & 4.5\end{array}$

$\begin{array}{llllllllll}0.95 & 0.9 & 0.8 & 0.7 & 0.6 & 0.5 & 0.4 & 0.3 & 0.2 & 0.1\end{array}$

$\begin{array}{llllll}50 & 60 & 70 & 80 & 90 & 100\end{array}$

1
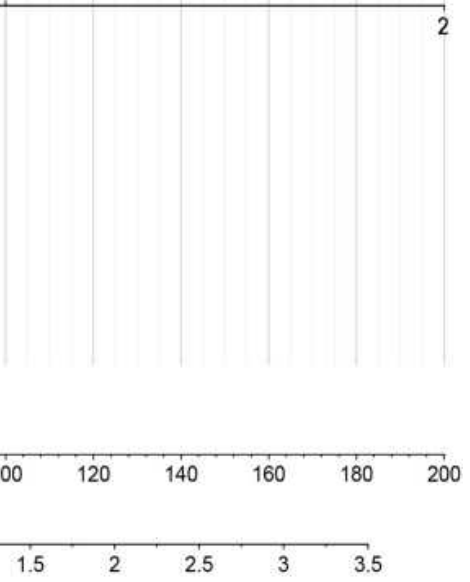

(1)


A

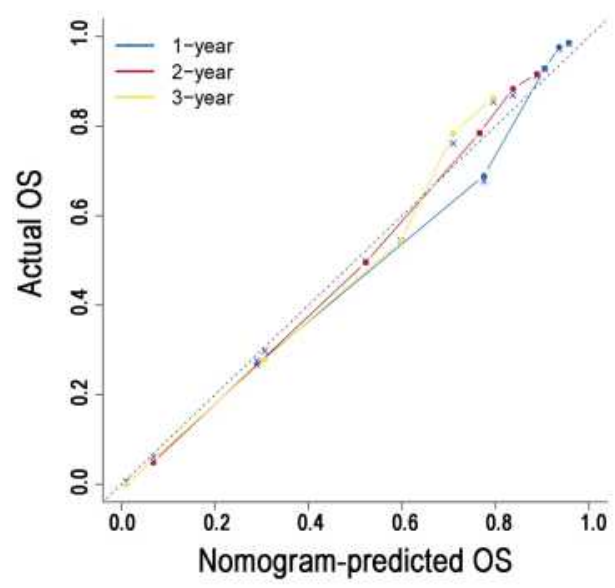

C

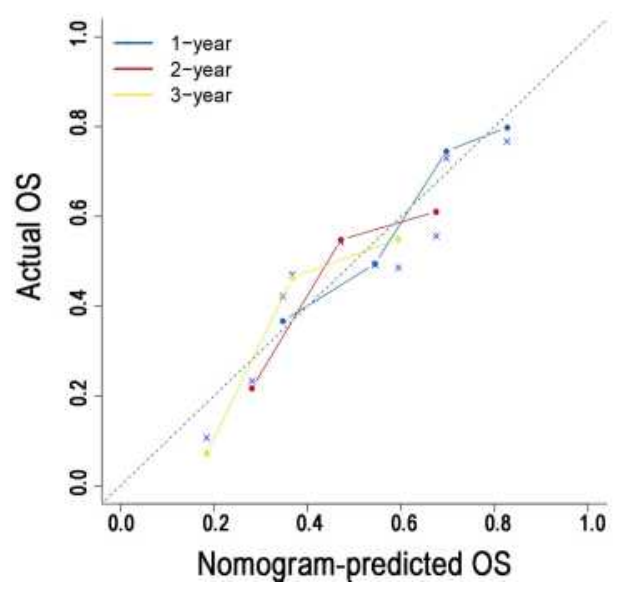

B

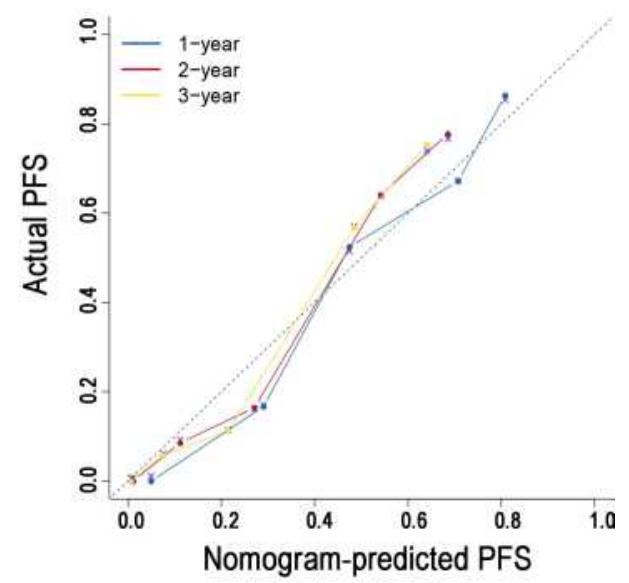

D

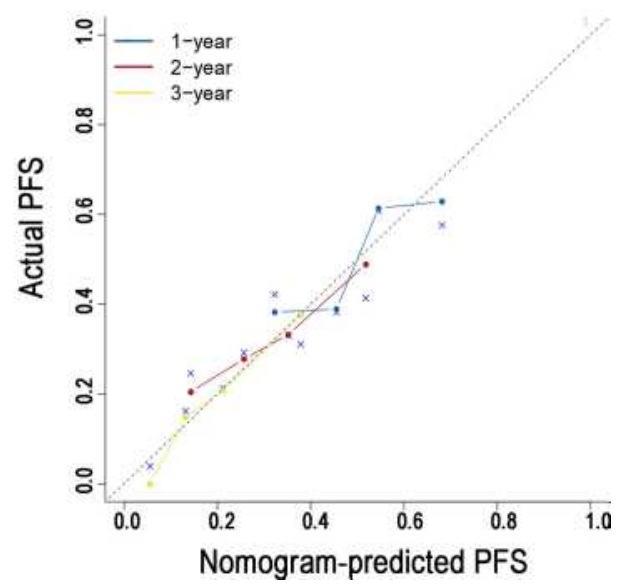

Figure 4 The calibration curve for predicting patient survival at I-, 2- and 3-year OS and PFS in the primary (A and B) and validation (C and D) cohorts, respectively.

The survival analysis of inflammation-based indexes had indicated that mGPS, which was the combination of C-reactive protein (CRP) and albumin (ALB), was an independent prognostic factor of survival. ${ }^{23} \mathrm{CRP}$ was a typical protein produced by hepatocytes or induced by cytokines, such as IL-6, while ALB was produced by liver and could reflect the nutritional status of patients. ${ }^{24}$ Previous study had shown that changes in the quantity and levels of alkaline phosphatase (ALP), CRP and ALB played a dominant role in the inflammatory response, triggered by harmful stimulation and conditions, including infection, injury and tissue malfunction. ${ }^{25,26}$ Similar with previous studies, ${ }^{26,27}$ higher levels of ALP indicated poorer survival of patients. Additionally, increasing evidences have indicated the pivotal role of CRP in tumor development. Apart from the higher levels of CRP identified T-lymphocyte impairment, the elevation of CRP levels was also directly associated with the circulating concentrations of vascular endothelial growth factor, allowing unrestrained tumor growth and dissemination. ${ }^{28,29}$ Additionally, there was a closed relationship between the levels of CRP and ALB. It was observed that the ALB levels decreased as circulating CRP levels increased. ${ }^{30}$ The elevated levels of CRP to more than $10 \mathrm{mg} / \mathrm{mL}$, combined with the levels of ALB decreased to less than $32 \mathrm{~g} / \mathrm{L}$ indicated the highest HRs on survival. ${ }^{31}$ Therefore, similar with other studies, ${ }^{32,33}$ two key inflammation components, CRP and ALB contributed to the significantly higher predictive power of mGPS, compared with other inflammation-based indexes. These 
Table 2 The Comparisons of ROC and C-Index Values in Primary and Validation Cohorts

\begin{tabular}{|c|c|c|c|c|c|c|c|c|c|c|c|}
\hline \multicolumn{2}{|c|}{ Stage } & \multicolumn{5}{|c|}{ Primary Cohort } & \multicolumn{5}{|c|}{ Validation Cohort } \\
\hline & & \multicolumn{3}{|c|}{ AUC } & \multirow[t]{2}{*}{ C-Index } & \multirow[t]{2}{*}{$P$ value } & \multicolumn{3}{|c|}{ AUC } & \multirow[t]{2}{*}{ C-Index } & \multirow[t]{2}{*}{$P$ value } \\
\hline & & I-Year & 2-Year & 3-Year & & & I-Year & 2-Year & 3-Year & & \\
\hline \multirow[t]{5}{*}{ OS } & Nomogram & 0.875 & 0.844 & 0.844 & $\begin{array}{l}0.800 \\
(0.767-0.833)\end{array}$ & Reference & 0.614 & 0.664 & 0.631 & $\begin{array}{l}0.659 \\
(0.586-0.732)\end{array}$ & Reference \\
\hline & TNM & 0.582 & 0.584 & 0.595 & $\begin{array}{l}0.569 \\
(0.528-0.610)\end{array}$ & $<0.001$ & 0.455 & 0.421 & 0.440 & $\begin{array}{l}0.553 \\
(0.497-0.609)\end{array}$ & 0.003 \\
\hline & $\mathrm{BCLC}$ & 0.693 & 0.679 & 0.715 & $\begin{array}{l}0.649 \\
(0.611-0.687)\end{array}$ & $<0.001$ & 0.463 & 0.469 & 0.544 & $\begin{array}{l}0.529 \\
(0.462-0.596)\end{array}$ & $<0.001$ \\
\hline & CLIP & 0.639 & 0.648 & 0.619 & $\begin{array}{l}0.599 \\
(0.557-0.64 I)\end{array}$ & $<0.001$ & 0.464 & $0.47 I$ & 0.548 & $\begin{array}{l}0.528 \\
(0.462-0.594)\end{array}$ & $<0.001$ \\
\hline & Okuda & 0.519 & 0.516 & 0.520 & $\begin{array}{l}0.511 \\
(0.491-0.531)\end{array}$ & $<0.001$ & 0.551 & 0.528 & 0.495 & $\begin{array}{l}0.546 \\
(0.479-0.612)\end{array}$ & $<0.001$ \\
\hline \multirow[t]{5}{*}{ PFS } & Nomogram & 0.806 & 0.810 & 0.799 & $\begin{array}{l}0.752 \\
(0.718-0.786)\end{array}$ & Reference & 0.580 & 0.590 & 0.519 & $\begin{array}{l}0.638 \\
(0.57 I-0.705)\end{array}$ & Reference \\
\hline & TNM & 0.589 & 0.615 & 0.617 & $\begin{array}{l}0.575 \\
(0.54 I-0.609)\end{array}$ & $<0.001$ & 0.448 & 0.436 & 0.463 & $\begin{array}{l}0.542 \\
(0.491-0.593)\end{array}$ & 0.002 \\
\hline & $\mathrm{BCLC}$ & 0.675 & 0.656 & 0.655 & $\begin{array}{l}0.613 \\
(0.580-0.646)\end{array}$ & $<0.001$ & 0.508 & 0.490 & 0.497 & $\begin{array}{l}0.496 \\
(0.436-0.556)\end{array}$ & $<0.001$ \\
\hline & CLIP & 0.661 & 0.643 & 0.636 & $\begin{array}{l}0.600 \\
(0.566-0.634)\end{array}$ & $<0.001$ & 0.507 & 0.491 & 0.500 & $\begin{array}{l}0.504 \\
(0.444-0.564)\end{array}$ & $<0.001$ \\
\hline & Okuda & 0.512 & 0.515 & 0.511 & $\begin{array}{l}0.503 \\
(0.489-0.517)\end{array}$ & $<0.001$ & $0.54 I$ & 0.487 & 0.432 & $\begin{array}{l}0.532 \\
(0.469-0.595)\end{array}$ & $<0.001$ \\
\hline
\end{tabular}

Abbreviations: OS, overall survival; PFS, progression-free survival; AUC, area under the receiver-operating characteristic; C-index, concordance index; TNM, tumor-nodemetastasis; BCLC, Barcelona Clinic Liver Cancer; CLIP, Cancer of the Liver Italian Program.

results provided insight into the relationship between inflammation and survival, serving the purpose of detailing prognostic information of mGPS.

In terms of pathological factors, increasing tumor numbers and tumor size, indicating large tumor burden, were associated with poor OS and PFS in this study. Additionally, imaging tumor size was also identified as a significantly prognostic factor for PFS. However, compared with pathological tumor size, imaging tumor size weighted less in PFS prediction. The calculation of pathological tumor size was much more objective while the imaging one was slightly more subjective, because the boundary of tumor might be indistinct on images. This may explain the greater role of pathological tumor size in survival prediction than the imaging one. LN metastasis was also an important prognostic factor for OS while it was failed to independently predict PFS. Oppositely, chemotherapy was shown to be a prognostic factor for PFS in this study. Patients with LN metastasis were classified as advanced tumor stages in TNM system and these patients would be often recommended to receive chemotherapy in clinical practice. Considering the large predictive power of chemotherapy in PFS prediction, the significance of LN metastasis may be overwhelmed by chemotherapy in the survival analysis of PFS in this study. Also, the survival discrepancy of LN metastasis can also be due to the selection bias in this retrospective study and more insights concerning the prognostic impact of LN metastasis are needed from prospective studies. Apart from pathological factors, CA19-9 and CEA were both indicators of survival outcome. Similar with previous study, ${ }^{34,35}$ CA19-9 and CEA, reflecting the tumor burden and activity, represent the most frequently used clinical prognostic factors in ICC patients. Increasing levels of CA19-9, combined with CEA, was shown to predict poor survival of patients with ICC after surgical resection. ${ }^{34}$

Several studies for predicting survival of ICC patients after surgery had been reported before. ${ }^{36,37}$ Compared with these studies, our study has several advantages. Firstly, as a study based on multiple cohorts, the number of included patients in this study was larger than those of other studies. Additionally, a total of 82 high-dimensional radiological 

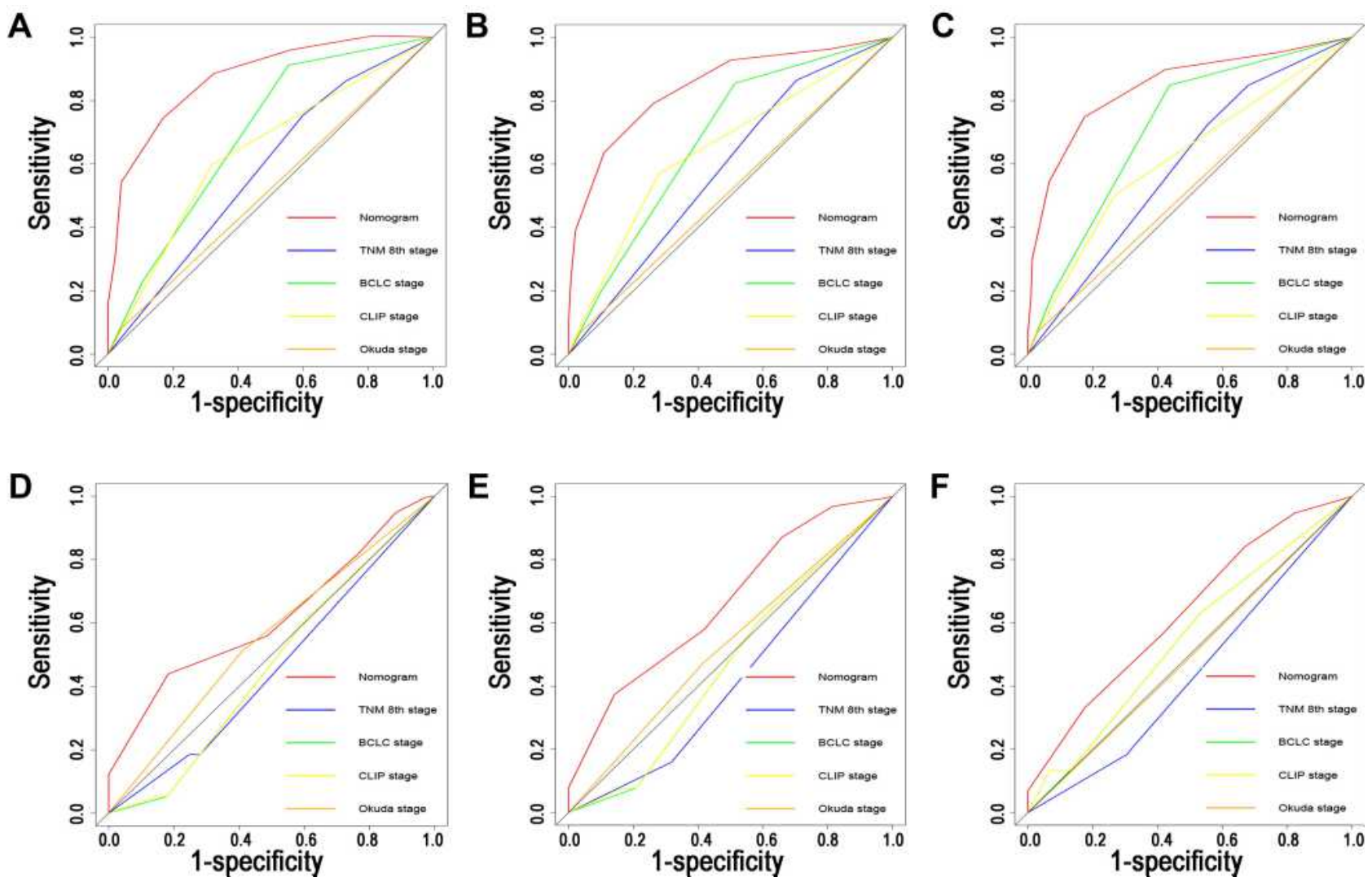

Figure 5 Comparisons of ROC curves of the nomogram, TNM staging system, BCLC staging score, CLIP score, and Okuda staging system for I-, 2- and 3-year OS in the primary (A-C) and validation (D-F) cohorts.

Abbreviations: ROC, receiver operating characteristic; TNM, tumor-node-metastasis; BCLC, Barcelona Clinic Liver Cancer; CLIP, Cancer of the Liver Italian Program; OS, overall survival.

and pathological data were analyzed in this study. The data amount was significantly larger than that in other studies. Thirdly, in terms of the key prognostic factor identified for OS and PFS, best predictive performance was selected by LASSO-penalized Cox regression analyses, whose values of C-index and AUC were significantly higher. Additionally, compared with traditional staging systems, including TNM, BCLC, CLIP, and Okuda stages, the established nomograms relied on not only common pathological factors or tumor markers, but also inflammationbased indexes, which were shown to have great predictive power for survival. The inclusion of specific indicators of survival in addition to pathological characteristics of primary tumor ensured that the established nomograms would display better discrimination power. The significantly higher values of AUC and C-indexes just indicated the better predictive power in survival prediction of nomograms, compared with other staging systems. Additionally, the established nomograms were well-validated in both primary and validation cohorts and showed significantly better predictive power in both cohorts. The relatively large size of the study cohorts in this study could make these results more generalizable. Furthermore, patients with higher nomogram-based scores had significantly lower survival rates than those with lower scores, illustrating the nomograms could stratify patients with ICC to subgroups with different survival after resection. Therefore, these newly established nomograms can be used as practical tools to predict survival of patients with ICC after surgical resection and have the potential for use in decision-making regarding the subsequent treatment for ICC patients following surgery. Apart from the precise prediction of survival rates after resection, the nomograms also indicated that patients with high nomogram-based scores needed to receive specific adjuvant therapy as soon as possible after resection to obtain a better survival.

This study has several limitations. Firstly, our efforts were limited by the retrospective nature of this study. Further largecohort prospective studies are needed to consolidate the results of this study. Secondly, the specific period and regimen of chemotherapy could impact survival of patients while they were not included in this study. Finally, although significantly 

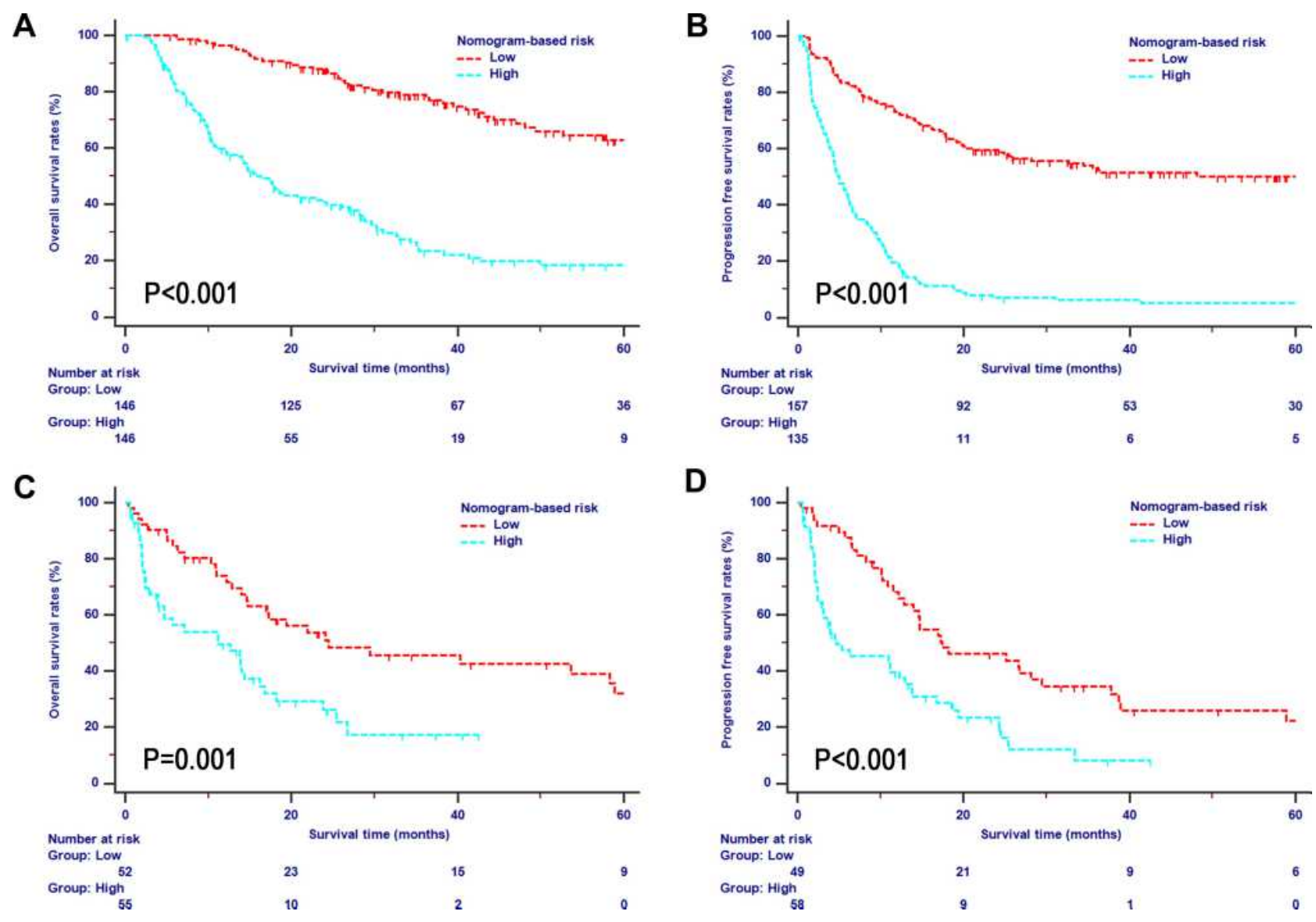

D

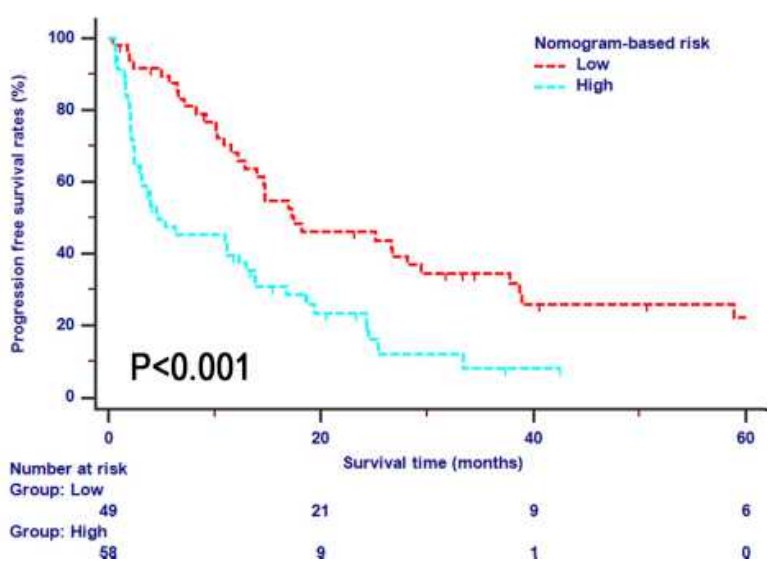

Figure 6 OS and PFS analyses stratified by nomogram-based risk scores of ICC patients after resection in the primary (A and B) and validation (C and D) cohorts. Abbreviations: OS, overall survival; PFS, progression-free survival; ICC, intrahepatic cholangiocarcinoma.

higher values of C-indexes and AUC of the established nomograms were shown for the validation of the nomograms, these relative values were not high enough. More validations based on other independent cohorts are necessary for the further use of the established nomograms.

\section{Conclusion}

In conclusion, we analyzed the prognostic value of clinicopathological factors in ICC patients after resection and built specific inflammation index-based nomograms for survival prediction. The established nomograms were well-calibrated and showed better predictive power than other staging systems. They could be used to help doctors to provide highly tailored management in ICC patient after resection in clinical practice.

\section{Acknowledgments}

This study was supported by the funding of Guangdong Basic and Applied Basic Research Foundation (2020A1515110954) and Sun Yat-sen University Grant for Medical Humanities Practice and Teaching (No. 23000-18008023).

\section{Disclosure}

The authors declare that they have no conflicts of interest for this work.

\section{References}

1. Siegel RL, Miller KD, Jemal A. Cancer statistics, 2020. CA Cancer J Clin. 2020;70(1):7-30. doi:10.3322/caac. 21590

2. Saha SK, Zhu AX, Fuchs CS, Brooks GA. Forty-year trends in cholangiocarcinoma incidence in the U.S.: intrahepatic disease on the rise. Oncologist. 2016;21(5):594-599. doi:10.1634/theoncologist.2015-0446

3. Banales JM, Cardinale V, Carpino G, et al. Expert consensus document: cholangiocarcinoma: current knowledge and future perspectives consensus statement from the European Network for the Study of Cholangiocarcinoma (ENS-CCA). Nat Rev Gastroenterol Hepatol. 2016;13(5):261-280.

4. Mavros MN, Economopoulos KP, Alexiou VG, Pawlik TM. Treatment and prognosis for patients with intrahepatic cholangiocarcinoma: systematic review and meta-analysis. JAMA Surg. 2014;149(6):565-574. doi:10.1001/jamasurg.2013.5137 
5. Prospective validation of the CLIP score: a new prognostic system for patients with cirrhosis and hepatocellular carcinoma. The Cancer of the Liver Italian Program (CLIP) investigators. Hepatology. 2000;31(4):840-845. doi:10.1053/he.2000.5628

6. Adhoute X, Penaranda G, Raoul JL, et al. Usefulness of staging systems and prognostic scores for hepatocellular carcinoma treatments. World J Hepatol. 2016;8(17):703-715. doi:10.4254/wjh.v8.i17.703

7. Edge SB, Byrd DR, Comptom CC, Fritz AG, Greene FL, Trotti A, eds. AJCC Cancer Staging Manual. 7th ed. New York, NY: Springer; 2010.

8. Colotta F, Allavena P, Sica A, Garlanda C, Mantovani A. Cancerrelated inflammation, the seventh hallmark of cancer: links to genetic instability. Carcinogenesis. 2009;30(7):1073-1081. doi:10.1093/carcin/bgp 127

9. Zhang H, Ren D, Jin X, Wu H. The prognostic value of modified Glasgow prognostic score in pancreatic cancer: a meta-analysis. Cancer Cell Int. 2020;20(1):462. doi:10.1186/s12935-020-01558-4

10. Sun S, He C, Wang J, Huang X, Wu J, Li S. The prognostic significance of inflammation-based scores in patients with ampullary carcinoma after pancreaticoduodenectomy. BMC Cancer. 2020;20 (1):981. doi:10.1186/s12885-020-07482-0

11. Han D, Zhang J, Zhao J, et al. Platelet-to-lymphocyte ratio is an independent predictor of chemoradiotherapy-related esophageal fistula in esophageal cancer patients. Ann Transl Med. 2020;8(18):1163. doi:10.21037/atm-20-4053

12. Salcedo MP, Sood AK, Dos Reis R, et al. Perineural invasion (PNI) in vulvar carcinoma: a review of 421 cases. Gynecol Oncol. 2019;152 (1):101-105. doi:10.1016/j.ygyno.2018.10.035

13. He C, Huang X, Zhang Y, et al. Signature for predicting recurrence risk of pancreatic ductal adenocarcinoma after radical resection. Front Oncol. 2019;9:1197. doi:10.3389/fonc.2019.01197

14. de Jong MC, Nathan H, Sotiropoulos GC, et al. Intrahepatic cholangiocarcinoma: an international multi-institutional analysis of prognostic factors and lymph node assessment. J Clin Oncol. 2011;29 (23):3140-3145. doi:10.1200/JCO.2011.35.6519

15. Harrell FE Jr, Lee KL, Mark DB. Multivariable prognostic models: issues in developing models, evaluating assumptions and adequacy, and measuring and reducing errors. Stat Med. 1996;15(4):361-387. doi:10.1002/ (SICI)1097-0258(19960229)15:4<361::AID-SIM168>3.0.CO;2-4

16. Pencina MJ, D’Agostino RB. Overall C as a measure of discrimination in survival analysis: model specific population value and confidence interval estimation. Stat Med. 2004;23(13):2109-2123. doi: $10.1002 / \operatorname{sim} .1802$

17. He CB, Lao XM, Lin XJ. Transarterial chemoembolization combined with recombinant human adenovirus type $5 \mathrm{H} 101$ prolongs overall survival of patients with intermediate to advanced hepatocellular carcinoma: a prognostic nomogram study. Chin J Cancer. 2017;36 (1):59. doi:10.1186/s40880-017-0227-2

18. Tang Z, Liu WR, Zhou PY, et al. Prognostic value and predication model of microvascular invasion in patients with intrahepatic cholangiocarcinoma. J Cancer. 2019;10(22):5575-5584. doi:10.7150/ jca.32199

19. Jayaramayya K, Balachandar V, Santhy KS. Ampullary carcinoma-a genetic perspective. Mutat Res. 2018;776:10-22.

20. Boonstra K, Weersma RK, van Erpecum KJ, et al. Population-based epidemiology, malignancy risk, and outcome of primary sclerosing cholangitis. Hepatology. 2013;58(6):2045-2055. doi:10.1002/hep.26565

21. Branchi V, Jürgensen B, Esser L, et al. Tumor infiltrating neutrophils are frequently found in adenocarcinomas of the biliary tract and their precursor lesions with possible impact on prognosis. J Pers Med. 2021;11(3):233. doi:10.3390/jpm11030233
22. Høgdall D, O'Rourke CJ, Dehlendorff C, et al. Serum IL6 as a prognostic biomarker and IL6R as a therapeutic target in biliary tract cancers. Clin Cancer Res. 2020;26(21):5655-5667. doi:10.1158/ 1078-0432.CCR-19-2700

23. McMillan DC, Crozier JE, Canna K, Angerson WJ, McArdle CS. Evaluation of an inflammation-based prognostic score (GPS) in patients undergoing resection for colon and rectal cancer. Int $J$ Colorectal Dis. 2007;22(8):881-886. doi:10.1007/s00384-006-0259-6

24. Pepys MB, Hirschfield GM. C-reactive protein: a critical update. J Clin Invest. 2003;111(12):1805-1812. doi:10.1172/JCI200318921

25. Medzhitov R. Origin and physiological roles of inflammation. Nature. 2008;454(7203):428-435. doi:10.1038/nature07201

26. Li Y, Wang JS, Guo Y, Zhang T, Li LP. Use of the alkaline phosphatase to prealbumin ratio as an independent predictive factor for the prognosis of gastric cancer. World J Gastroenterol. 2020;26 (44):6963-6978. doi:10.3748/wjg.v26.i44.6963

27. Liu X, Hou Y, Wang X, et al. Machine learning-based development and validation of a scoring system for progression-free survival in liver cancer. Hepatol Int. 2020;14(4):567-576. doi:10.1007/s12072020-10046-w

28. Kemik O, Kemik AS, Begenik H, et al. The relationship among acute-phase response proteins, cytokines, and hormones in various gastrointestinal cancer types patients with cachectic. Hum Exp Toxicol. 2012;31(2):117-125. doi:10.1177/0960327111417271

29. Reynés G, Vila V, Martín M, et al. Circulating markers of angiogenesis, inflammation, and coagulation in patients with glioblastoma. J Neurooncol. 2011;102(1):35-41. doi:10.1007/ s11060-010-0290-x

30. McMillan DC, Elahi MM, Sattar N, Angerson WJ, Johnstone J, McArdle CS. Measurement of the systemic inflammatory response predicts cancer-specific and non-cancer survival in patients with cancer. Nutr Cancer. 2001;41(1-2):64-69. doi:10.1080/01635581.2001.9680613

31. Howren MB, Lamkin DM, Suls J. Associations of depression with C-reactive protein, IL-1, and IL-6: a meta-analysis. Psychosom Med. 2009;71(2):171-186. doi:10.1097/PSY.0b013e3181907c1b

32. Tong T, Guan Y, Xiong H, Wang L, Pang J. A meta-analysis of Glasgow prognostic score and modified Glasgow prognostic score as biomarkers for predicting survival outcome in renal cell carcinoma. Front Oncol. 2020;10:1541. doi:10.3389/fonc.2020.01541

33. Fujiwara R, Takemura K, Fujiwara M, et al. Modified Glasgow prognostic score as a predictor of prognosis in metastatic renal cell carcinoma treated with nivolumab. Clin Genitourin Cancer. 2020. doi:10.1016/j.clgc.2020.10.007

34. He C, Zhang Y, Song Y, et al. Preoperative CEA levels are supplementary to CA19-9 levels in predicting prognosis in patients with resectable intrahepatic cholangiocarcinoma. J Cancer. 2018;9 (17):3117-3128. doi:10.7150/jca.25339

35. Yoo T, Park SJ, Han SS, et al. Postoperative CA19-9 change is a useful predictor of intrahepatic cholangiocarcinoma survival following liver resection. Dis Markers. 2015;2015:298985. doi:10.1155/ 2015/298985

36. Matsumoto $\mathrm{T}$, Itoh S, Yoshizumi $\mathrm{T}$, et al. C-reactive protein: albumin ratio in patients with resectable intrahepatic cholangiocarcinoma. BJS Open. 2020;4(6):1146-1152. doi:10. $1002 /$ bjs 5.50348

37. Lu LH, Zhong C, Wei W, et al. Lymphocyte-C-reactive protein ratio as a novel prognostic index in intrahepatic cholangiocarcinoma: a multicentre cohort study. Liver Int. 2021;41(2):378-387. doi:10.1111/liv.14567 


\section{Publish your work in this journal}

The Journal of Inflammation Research is an international, peerreviewed open-access journal that welcomes laboratory and clinical findings on the molecular basis, cell biology and pharmacology of inflammation including original research, reviews, symposium reports, hypothesis formation and commentaries on: acute/chronic inflammation; mediators of inflammation; cellular processes; molecular mechanisms; pharmacology and novel anti-inflammatory drugs; clinical conditions involving inflammation. The manuscript management system is completely online and includes a very quick and fair peerreview system. Visit http://www.dovepress.com/testimonials.php to read real quotes from published authors. 